\title{
Synthesis of carbon nanotubes via chemical vapor deposition by using rare- earth metals as catalysts
}

\author{
Anna Jędrzejewska, Karolina Wnuk, Ryszard J. Kaleńczuk, Ewa Borowiak-Paleń \\ West Pomeranian University of Technology, Szczecin, Institute of Chemical and Environment Engineering, \\ ul. Pulaskiego 10, 70-322 Szczecin, Poland, corresponding author: e-mail: eborowiak@zut.edu.pl
}

\begin{abstract}
This work presents the results of the synthesis of carbon nanotubes using the CVD method. Fe:MgO catalyst was used, also in combination with rare earth elements (gadolinium (Gd), dysprosium (Dy)), which when used alone, are not efficient as catalysts in nanotube growth. Synthesis was performed both at reduced pressure $\left(10^{-3} \mathrm{mbar}\right)$ and atmospheric pressure, with constant parameters dependent on the process parameters.
\end{abstract}

Keywords: carbon nanotubes, chemical vapor deposition CVD, Raman spectroscopy, Transmission Electron Microscopy.

\section{INTRODUCTION}

Inspired by the work by Krätschmer and Hofmann, Sumio Iijima initiated a detailed study on carbon black from arc sublimation of fullerenes, using Transmission Electron Microscopy (TEM). He studied the cathode deposit which, as it turned out, contained a new graphite structure characterized by long and thin fibers ${ }^{1}$. The discovery of new carbon nanomaterials boosted the interest in research on new crystalline forms of carbon.

Carbon nanotubes have unique chemical properties (chemical resistance), mechanical properties (high stiffness and mechanical strength) and electronic properties (high thermal and electrical conductivity) ${ }^{2,3,4}$.Therefore, their practical application in numerous sciences is under research 5 .

Since the discovery of carbon nanotubes, many methods have been described to produce them. They include: the electric arc method ${ }^{6}$, catalytic laser synthesis ${ }^{7}$, and various techniques of chemical vapor deposition (CVD) ${ }^{8}$. The most interesting method, due to the possibility to keep the process temperature relatively low, with high process efficiency and purity, producing carbon nanotubes on an industrial scale, is the CVD method.

This paper presents the results of the synthesis of carbon nanotubes using the CVD method. Fe:MgO catalyst was used, also in combination with rare earth elements (gadolinium, dysprosium), which when used alone, are not efficient as catalysts to obtain nanotubes. Synthesis was performed both at reduced pressure $\left(10^{-3} \mathrm{mbar}\right)$ and atmospheric pressure, with constant parameters dependent on the reaction environment.

\section{EXPERIMENTAL}

Three catalyst types, Fe:MgO (molar ratio 1:25) ${ }^{9}$, Fe:Gd:MgO (1:0, 25:25) and Fe:Dy:MgO (1:0, 25:25), were used for the nanotube syntheses. Adequate catalyst precursors: iron (II) acetate $-\mathrm{Fe}\left(\mathrm{CO}_{2} \mathrm{CH}_{3}\right)_{2}$, gadolinium (III) acetate hydrate $-\mathrm{Gd}\left(\mathrm{CH}_{3} \mathrm{CO}_{2}\right)_{3} * \mathrm{xH}_{2} \mathrm{O}$ or dysprosium (III) acetate hydrate $-\mathrm{Dy}\left(\mathrm{CH}_{3} \mathrm{CO}_{2}\right)_{3} \cdot \mathrm{xH}_{2} \mathrm{O}$ were mixed with magnesium oxide $-\mathrm{MgO}$ at the right mix ratio by weight. Subsequently, butyl alcohol was added to the resulting mixture, and the total sample was dispersed for one hour in an ultrasound bath in order to reduce the size of larger particles in the mixture and to mix them thoroughly. Then, the catalytic solution was dried at $200^{\circ} \mathrm{C}$ in order to evaporate the butanol. The dry mass resulting from the evaporation of butanol was then grounded in a mortar to obtain a fine powdered catalyst.

The synthesis was performed both at reduced pressure (vacuum, $10^{-3} \mathrm{mbar}$ ), and at atmospheric pressure. The process temperature was $950^{\circ} \mathrm{C}$, and the process duration was 30 minutes. For reduced pressure synthesis, the partial pressure of carbon source (ethanol) was 55 mbar. During the synthesis in atmospheric conditions, the flow rate of inert gas (argon) was 0.61 per minute. Upon the end of the synthesis, the oven heating was turned off. After the cooling stage, the sample was removed and cleaned.

The raw material was placed in a beaker containing 35 $-38 \%$ hydrochloric acid. Then the entire material was subjected to ultrasound in a water bath for 60 minutes. Afterwards, the solution was filtrated using a set of a syringe and a special tip with a membrane lining inside, resistant to concentrated acids. The sample was washed with distilled water and acetone. With this purification method, the morphology of carbon nanotubes remains unchanged, permitting further analysis for the purposes of characterization.

The resulting nanotubes were characterized with the use of the following methods: Raman Spectroscopy (inVia Renishaw/785nm), Transmission Electron Microscopy (Tecnai F20 FEI), and thermogravimetry, with a thermobalance equipped with a differential thermal analyzer (DTA-Q 600 SDT TA Instruments).

\section{RESULTS AND DISCUSSION}

The obtained materials were examined by three different analytical methods.

The use of Raman Spectroscopy in order to identify the reaction products showed that depending on the reaction environment, three types of nanotubes were obtained: single-walled carbon nanotubes (SWCNT), double-walled carbon nanotubes (DWCNT), and multi-walled carbon nanotubes (MWCNT). In order to validate the results, Transmission Electron Microscopy and Thermogravimetry Analysis were carried out. Using Raman spectra, the purity of the produced nanotubes was assessed, and the range of diameters of the carbon nanotubes was specified on the 
basis of Transmission Electron Microscopy images. Using the thermogravimetry, the content of amorphous carbon, carbon nanotubes and other impurities was determined in the samples after the synthesis

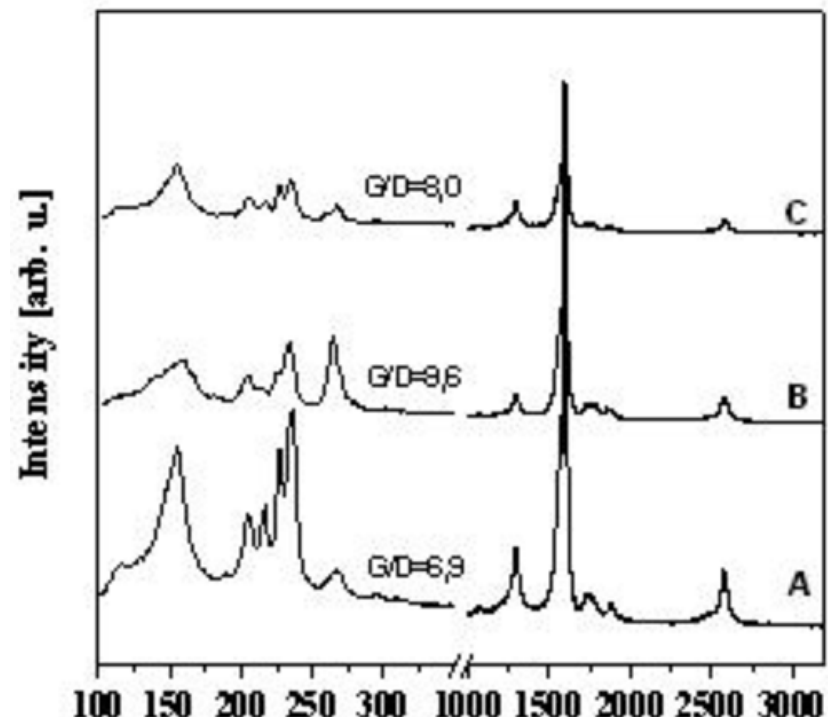

Raman shift $\left[\mathrm{c} \mathrm{m}^{-1}\right]$

Figure 1. Raman spectra of carbon nanotubes produced by reduced pressure used the catalyst $\mathrm{A}-\mathrm{Fe}: \mathrm{MgO}$, B - Fe:Dy:MgO, C - Fe:Gd:MgO

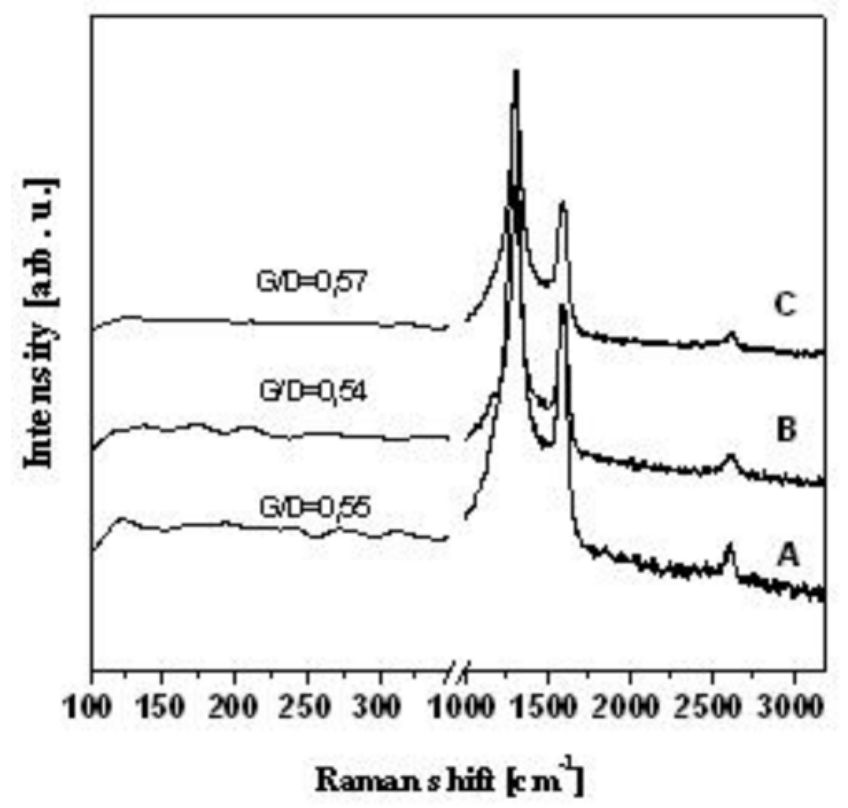

Figure 2. Raman spectra of carbon nanotubes produced by atmospheric pressure used the catalyst A- Fe:MgO, B - Fe:Dy:MgO, C - Fe:Gd:MgO

Based on the Raman spectra, the type of resulting carbon nanotubes can be identified, which is demonstrated by peaks in $100-300 \mathrm{~cm}^{-1}$ frequency ranges (RBM peak - normal "breathing" vibration), 1250 - $1450 \mathrm{~cm}^{-1}$ (D peak - defects, flaws in the crystal structure of graphite) and $1542-1634 \mathrm{~cm}^{-1}$ (G peak - graphitic structures), $2500-2900 \mathrm{~cm}^{-1}$ ( $\mathrm{G}^{\prime}$ peak - photon resonance). The presence of the RBM peak shows that the samples can be made of single- or double-walled carbon nanotubes. Sample purity can be obtained from the $D$ peak to $G$ peak ratio.
Single- and double-walled carbon nanotubes were produced by reduced pressure synthesis (Fig. 1), and multiwalled carbon nanotubes resulted from reactions at atmospheric pressure (no RBM peaks present, G, D and G' peaks observed) (Fig. 2). However, Figure 2 shows that Fe:MgO and Fe:Gd:Mgo catalysts give MWCNT with iron nanowires being confined in the graphitic segments along the tube. These structures exhibit bamboo like morphology. Applocation of catalyst Fe:Dy:MgO in CVD process results in hollow MWCNT with many defects in the sidewalls. As regards the samples obtained at reduced pressure, the most defective sample was generated with the use of the Fe:MgO catalyst, and the least defective with the use of the Fe:Dy: $\mathrm{MgO}$ catalyst. At atmospheric pressure, the most defective sample used Fe:Dy:MgO catalyst, and the least defective sample used Fe:Gd:MgO catalyst.

Difference in the morphology obtained in two presented experimental setups can be due to much higher concentration of carbon source available for the nanotube formation $^{10}$.

By analyzing TEM images, the resulting nanotube types were confirmed. The distribution of nanotube diameters was calculated on the basis of TEM images. The diameters of nanotubes obtained at reduced pressure with $\mathrm{MgO}$ catalyst range from $1 \mathrm{~nm}$ to $3 \mathrm{~nm}$, with Fe: Gd:MgO catalyst from $1 \mathrm{~nm}$ to $4 \mathrm{~nm}$, and with Fe:Gd:MgO catalyst from $1 \mathrm{~nm}$ to $5 \mathrm{~nm}$. The diameters of nanotubes obtained at atmospheric pressure with $\mathrm{Fe}: \mathrm{Gd}: \mathrm{MgO}$ catalyst range from $16 \mathrm{~nm}$ to $41 \mathrm{~nm}$, with Fe:MgO catalyst from $23 \mathrm{~nm}$ to $68 \mathrm{~nm}$, and with Fe:Dy:MgO catalyst from $17 \mathrm{~nm}$ to 39 $\mathrm{nm}$.

In order to determine the exact content of carbon nanotubes, amorphous carbon and other residual impurities, TGA analysis was carried out after the purification of the sample.

Analysis of the thermogravimetric curves (not presented in this paper) shows that the purest nanotubes are obtained with Fe:Dy:MgO catalyst at reduced pressure and $\mathrm{Fe}: \mathrm{MgO}$ catalyst at atmospheric pressure. Synthesis carried out at atmospheric pressure is characterized with higher carbon nanotube yield than at reduced pressure. At reduced pressure, the resulting material contains more amorphous carbon than the samples produced at atmospheric pressure.

It was determined that with the use of catalysts made of iron compounds with rare earth metals (Gd, Dy) deposited on magnesium oxide, maintaining the adequate mole ratio and based on the CVD method, carbon nanotubes were produced. The ethanol flow pattern during the synthesis governs the type of the resulting material. At reduced pressure, with the partial pressure of ethanol at 55 mbar, SWCNT and DWCNT were obtained, while in the presence of argon, with ethanol heated to $95^{\circ} \mathrm{C}, \mathrm{MWCNT}$ were produced. This offers the possibility to produce a selected type of nanotubes with the use of the same catalyst by adjusting the process conditions. The resulting multi-walled nanotubes could be used in the future as nanoreactors or nanocontainers to store and "carry" various elements and their compounds. In addition, nanotubes filled with a ferromagnetic material can serve as contrasting materials for the purposes of medical diagnostics and gene therapy, and could be used as drug carriers ${ }^{11-13}$. 

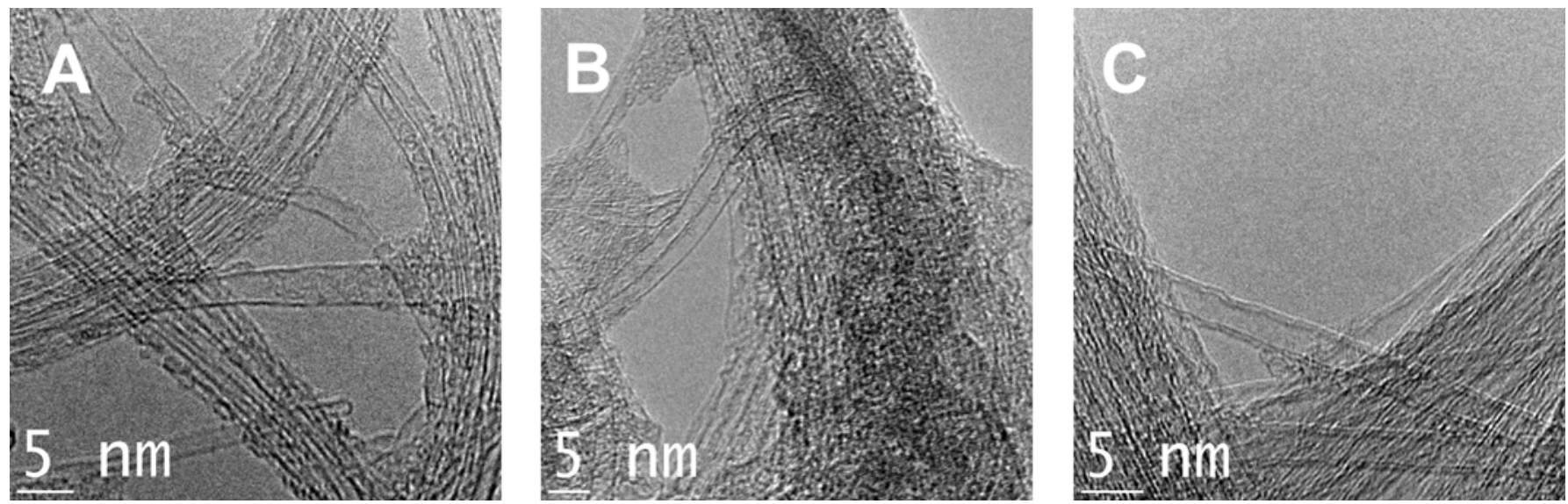

Figure 3. TEM images of carbon nanotubes synthesized by reduced pressure used the catalyst $\mathrm{A}-\mathrm{Fe}: \mathrm{MgO}, \mathrm{B}-\mathrm{Fe}: \mathrm{Dy}: \mathrm{MgO}$, $\mathrm{C}-\mathrm{Fe}: \mathrm{Gd}: \mathrm{MgO}$
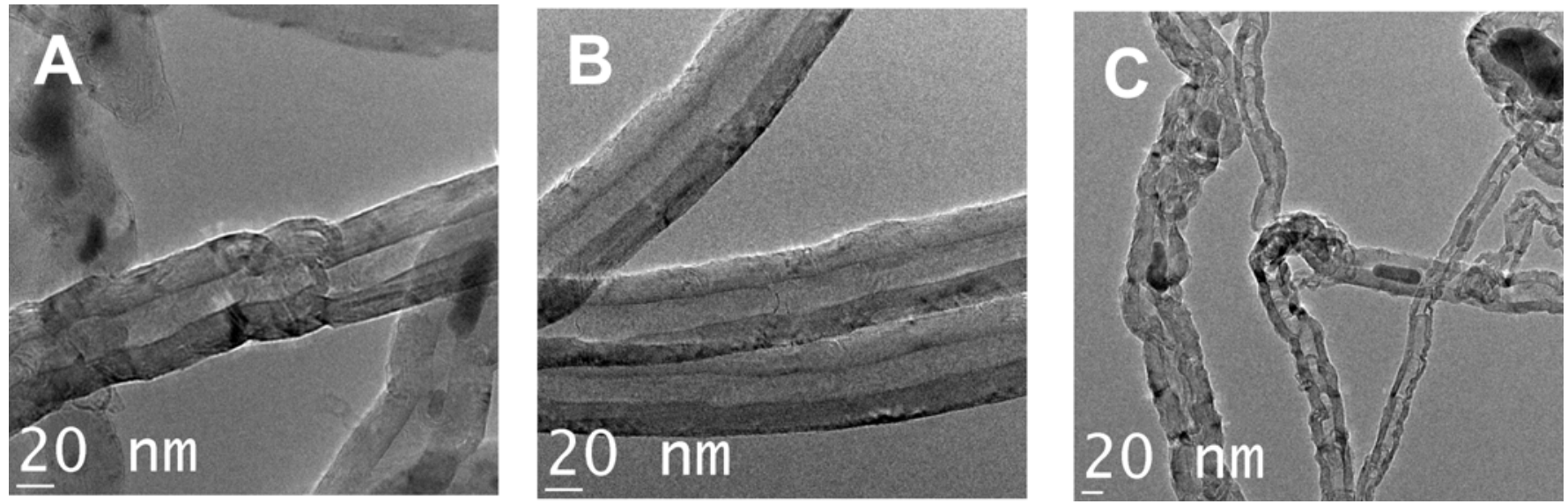

Figure 4. TEM images of carbon nanotubes synthesized by atmospheric pressure used the catalyst A - Fe:MgO, B - Fe:Dy:MgO, C - Fe:Gd:MgO

\section{CONCLUSION}

To conclude, ethanol with Fe:MgO, Fe:Gd:MgO and Fe:Dy:MgO catalysts forms a system permitting the production of single- and double-walled nanotubes with high efficiency and purity, while the argon atmosphere produces multi-walled nanotubes with high efficiency yet a high level of defects. This is an evidence that atmospheric pressure experment providing higher concentration of carbon feedstock leads to the formation of multiwalled carbon structure. However, this conditions are oxygen richer in comparison to the conditions present in reduced pressure experiments and this etches the graphite forming the tubes leading to the higher concentration of defects. Additionally, this is a first report on the efficiency of $\mathrm{Gd}$ and Dy with iron supported on $\mathrm{MgO}$ to produce carbon nanostructures. Application of those elements yields in enhancement of sample purities (Fe:Dy: $\mathrm{MgO}$ in low pressure CVD and Fe:Gd:MgO in atmospheric) in respect to the $\mathrm{Fe}: \mathrm{MgO}$. This is a key point to enhance the purity and quality of bulk scale samples widely produced using only iron as a catalyst.

\section{Acknowledgements}

This work was sponsored by Polish State Committee for Scientific Research grant 503- 10 - 011- 2404/4p (E-BP).

\section{LITERATURE CITED}

1. Iijima, S. (1991). Helical microtubules of graphitic carbon. Nature. 354, 56 - 58. DOI: 10.1038/354056a0.
2. Treacy, M.M.J., Ebbesen, T.W. \& Gibson, J.M. (1996). Exceptionally high Young's modulus observed for individual carbon nanotubes. Nature. 381, 678 - 680. DOI: 10.1038/ $381678 \mathrm{a} 0$.

3. Wong, E.W., Sheehan, P.E. \& Lieber, C.M. (1997). Nanobeam Mechanics: Elasticity, Strength, and Toughness of Nanorods and Nanotubes. Science. 26, 1971 - 1975 DOI: 10.1126/science.277.5334.1971.

4. Frank, S., Poncharal, P. Wang, Z.L. \& De Heer, W.A.(1998). Carbon Nanotube Quantum Resistors. Science. 280, 1744-1746. DOI: 10.1126/science.280.5370.1744.

5. Gong, Q., Li, Z., Zhou, X., Wu, J., Wang, Y. \& Liang, J.(2005). Synthesis Synthesis and characterization of in situ grown carbon nanofiber/nanotube reinforced carbon/carbon composites. Carbon. 43, 2426 - 2429 DOI: 10.1016/ j.carbon.2005.04.024.

6. Ebbesen, T.W. \& Ajayan, P.M. (1992). Large-scale synthesis of carbon nanotubes. Nature. 358, 220 - 222 DOI: 10.1038/ $358220 \mathrm{a} 0$.

7. Thess, A., Lee, R., Nikolaev, P., Dai, H. J., Petit, P. \& Robert, J., et al.(1996). Crystalline Ropes of Metallic Carbon nanotubes. Science. 273, 483 - 487. DOI: 10.1126/science.273.5274.483.

8. Murakami, Y., Miyauchi, Y, Chiashi, S. \& Maruyama, S. (2003). Characterization of Single-Walled Carbon Nanotubes Catalytically Synthesized from Alcohol. Chem. Phys. Lett. 374, 53 DOI: 10.1016/S0009-2614(03)00687-0.

9. Borowiak-Palen, E., Bachmatiuk, A., Rümmeli, M.H., Costa, S. \& Kalenczuk, R.J. (2008). Modifying CVD synthesized carbon nanotubes via the carbon feed rate. Physica E. 40, 2227 - 2230 DOI:10.1016/j.physe.2007.10.105.

10. Steplewska, A., Jedrzejewski, R. \& Borowiak-Palen, E. (2008). Preperation and characterization of catalyst mix Fe- 
$\mathrm{Co} / \mathrm{MgO}$ for carbon nanotubes growth. Polish Journal of Chemical Technology. 10, 3 - 3. DOI: 10.2478/v10026-0080028-0.

11. Suh, W.H., Suslick, K.S., Stucky, G.D. \& Suh, Y.H. (2009). Nanotechnology, nanotoxicology, and neuroscience. Progress in Neurobiology. 87(3), 133 - 70. DOI: 10.1016/ j.pneurobio.2008.09.009.

12. Raffa, V., et al.(2008). Can the properties of carbon nanotubes influence their internalization by living cells? Carbon. 46(12), 1600 - 1610. DOI: 10.1016/j.carbon.2008.06.053.

13. Shvedova, A.A., et al.(2009). Mechanisms of pulmonary toxicity and medical applications of carbon nanotubes: Two faces of Janus? Pharmacology \& Therapeutics. 121 (2), 192 - 204. DOI: doi:10.1016/j.pharmthera.2008.10.009. 